\title{
An update on the vertebrate homeobox
}

\author{
GREGORY R. DRESSLER \\ Department of MOleCUlar CELl BIology, \\ Max Planck Institute of Biophysical ChemistRy, \\ 3400 GÖTTINGEN, FRG
}

Given the complexity of development and the multitude of differentiated cell types in higher eukaryotic organisms, one might expect that a large variety of proteins would be required to regulate specific developmental processes. However, if the differentiated state of a particular cell is specified not by a single protein but by a combination of factors, significantly fewer regulatory proteins are required to constitute a unique genetic program for a single cell type. Furthermore, if the expression level of a single factor, in combination with others, is a determinative event, an even smaller set of governing factors would suffice.

Thus considering the finite number of controlling factors, it is remarkable that more and more potential developmental and cellspecific factors contain homeoboxes. While the evidence that vertebrate homeobox genes encode transcription factors active during development is mostly circumstantial, several functionally defined tissue-specific transcription factors have also been found to contain homeobox domains.

\section{New classes of mouse homeoboxes}

Drosophila homeoboxes fall into various categories depending on the genes in which they are found ${ }^{1}$. The Antennapedia (Antp) homeobox is the prototype but other types include the engrailed (en), bicoid, caudal (cad), paired (prd), and even-skipped (eve) homeoboxes. Although at least 24 mouse genes contain an Antp-type homeobox and at least two genes have the en-type homeobox ${ }^{2.3}$, murine sequences homologous to other Drosopbila homeobox classes have not been isolated until recently.

A new mouse homeobox locus on chromosome 5, Hox 7, was defined recently in two independent reports. Using the Drosopbila muscle segment bomeobox ( $m s b$ ) sequence, a genomic cosmid library was screened and a homologous mouse sequence with $92 \%$ amino acid identity was identified ${ }^{4}$. Surprisingly; the same gene was found in a mouse cDNA library by an entirely different screening method. Hill et al. 5 isolated cDNA clones that hybridized to Hox 1.6 at low stringency but did not hybridize to Drosophila fushi tarazu ( $f t z$ ), which contains an Antp-type homeobox. The resulting clone, Hox 7.1, shares only $56 \%$ nucleotide identity with $\operatorname{Hox} 1.6$ and less than 50\% with Antp or ftz. Preliminary evidence indicates that there may be several related genes with the muscle segment bomeo, box; however it is not known if these genes map to the same locus.

Many mouse homeobox genes are expressed in ectoderm- and mesoderm-derived tissues in a region-specific manner during embryogenesis ${ }^{6}$. However, the expression of Hox 7.1 in neural crest cells, but not neural tube, at 9 days gestation is a unique feature of this gene. In addition, expression of Hox 7.1 in the first visceral arch and subsequent mandible and maxillary structures may indicate a function during cranial neural crest cell predetermination. Such predetermination is apparent in transplantation experiments in the chick, which indicate that neural crest cells are predisposed to a particular developmental fate before they migrate to their final destination?. Thus, a mechanism for predisposition must exist that defines the eventual fate of specific subsets of neural crest cells. Whether homeobox genes are involved in this neural crest predetermination is an intriguing possibility that certainly warrants further investigation.

A second new type of homeobox gene characterized recently is the mouse $C d x 1$ gene $^{8}$, which con- tains a homeobox homologous to the Drosophila caudal gene. In fact, $C d x 1$ shares many sequence similarities with caudal besides the homeobox. The expression of $C d x 1$ is unusual, relative to other mouse Hox genes, because it peaks relatively late in development, continues into adulthood, and is restricted to epithelial cells of the intestinal tract that are derived from embryonic endoderm. These intestinal epithelial cells consist of four major differentiated cell types, all derived from a single stem cell population that continuously renews the cells of the villi; thus it would be interesting to determine if the $c d x 1$ gene is restricted to a single differentiated cell type or if it is found only in the progenitor cells.

\section{Transcription factors with homeodomains}

The Drosophila en , eve $^{10}$ and Antp ${ }^{11}$ homeodomains bind DNA in a sequence-specific manner. Furthermore, evidence supporting the helix-turn-helix tertiary structure model was recently obtained with nuclear magnetic resonance ${ }^{12}$. Whether these data can be extrapolated to vertebrate $H o x$ genes remains to be shown. However, while many investigators are eager to prove that $H o x$ genes encode transcription factors, several groups studying known transcription activators have cloned and sequenced the respective genes and have found homeodomains

A protein that binds the immunoglobulin promoter octamer sequence, the human Oct- 2 protein, contains a homeodomain that is only $33 \%$ homologous to the consensus homeodomain but, more importantly, it retains the helix-turn-helix structural motif 13,14 . A three amino acid substitution in the recognition helix disrupts the binding of Oct-2 to the octamer sequence ${ }^{13}$. Two other 
homeodomain-containing vertebrate transcription factors have recently been identified: Oct-1 (Ref. $15)$ is another immunoglobulin octamer-binding protein, but unlike Oct-2, which is B cell specific, it is expressed ubiquitously; the second protein, Pit-1 (Ref. 16) or GHF-1 (Ref. 17), has been isolated from the rat pituitary gland, and specifically activates the growth hormone and prolactin genes in cells of the somatotropic and lactotropic lineages. The protein contains a homeodomain with 35\% amino acid identity to the Drosophila prd homeobox.

These studies clearly demonstrate that mammalian proteins with homeodomains recognize specific sequences and activate transcription. However, target promoter sequences that bind the vertebrate Hox genes containing the Antptype homeodomains have not been identified to date.

\section{Functional assays for mammalian Hox gene function}

Unlike Drosophila, mouse mutants for particular Hox genes have not been described and thus assigning a developmental function to these genes has proved difficult. Not deterred by lack of naturally occurring or chemically induced mutants, modern molecular biologists have begun to circumvent this problem with innovative transgenic approaches

The first report of modulated Hox gene expression in transgenic mice was recently published by Wolgemuth et al. ${ }^{18}$. Through an apparent gene dosage effect, the Hox 1.4 gene was overexpressed in certain embryonic structures. In particular, the mesenchyme of the developing intestinal tract, which normally expresses low levels of Hox 1.4, showed a dramatic increase in mRNA levels. The resultant adult phenotype resembled congenital megacolon, as characterized by an enlarged bowel and the inability to extrude feces. It was postulated that a deficiency of neural crest cells, which innervate the colon and generate the myenteric ganglia, would explain this developmental defect.

That neural crest cells are potential targets of Hox gene func- tion was also demonstrated by Balling et $a l^{19}$ in transgenic mice expressing Hox 1.1. By coupling the Hox 1.1 coding sequence to a ubiquitous promoter, ectopic expression during development was observed. Craniofacial malformations were apparent in transgenic animals expressing $H o x \quad 1.1$ in anterior structures that normally do not express the gene. In fact, the resulting phenotype, which included cleft secondary palate, nonfused pinnae of the outer ear, and open eyes at birth, was strikingly similar to retinoic acid embryopathy, a syndrome induced by the administration of retinoic acid during pregnancy. The implications of this study are twofold: (1) that aspects of neural crest cell maturation during craniofacial morphogenesis can be disturbed by the ectopic expression of a homeobox gene, and (2) that retinoic acid may induce Hox gene expression in vivo, as it does in vitro.

In the amphibian Xenopus laevis, perturbation of dorsal root ganglia and dorsal fins has been reported by Cho et al.20 after microinjection of antibodies against the XlHbox1 homeodomain protein. Interestingly, both dorsal fin mesenchyme and dorsal root ganglia are derived form neural crest cells. Furthermore, the defect appeared to be specific to anterior ganglia only. The data again sug gest that neural crest cells are at least one potential target for Hox gene function.

In addition to gain-of-function mutants, gene targeting in embryonic stem cells can potentially generate loss-of-function mutant animals. In a recent report by Zimmer and Gruss ${ }^{21}$, the Hox 1.1 gene was mutated in embryonic stem cells by homologous recombination. Although these stem cells could contribute to chimeric mice, it is not yet known if germ-line transmission will occur and, subsequently, if heterozygous mice are viable. The viability of mutant lines is essential in addressing gene function and may be problematic considering the variety of tissues that express a single Hox gene and the potential for pleiotropism. In fact, the problem of viability has already surfaced in the study of Balling et al., where it was reported that animals expressing the transgene, with one exception, died shortly after birth.

In conclusion, it appears that the numerical limit of homeoboxcontaining genes is slowly being approached, particularly for the Antp-type homeobox genes, as the rate of new gene isolation decreases. New genes cloned recently are more and more divergent from the prototype Antp homeodomain. It is likely that mouse homologs to other Drosopbila homeobox sequences, such as eve and bicoid, do exist; however, they probably do not represent a family as large as the murine Hox genes. Although new genes will continue to be characterized, the current emphasis clearly is on assigning functions to the many $H o x$ and Hox-related genes already described.

\section{Acknowledgements}

I thank Rudi Balling and Michael Kessel for sharing results prior to publication and for stimulating discussions.

\section{References}

1 Gehring, W.J. (1987) Science 236, 1245-1252

2 Dressler, G.R. and Gruss, P. (1988) Trends Genet. 4, 214-219

3 Wright, C.V.E., Cho, K.W.Y., Oliver, G. and De Robertis, E.M.(1989) Trends Biochem. Sci. 14, 52-56

4 Robert, B. et al. (1989) EMBOJ.8, 91-100

5 Hill, R.E. et al. (1989) Genes Det: 3. $26-37$

6 Holland, P.W.H. and Hogan, B.L.M (1988) Genes Dev. 2, 773-782

7 Noden, D.M. (1988) Development 103 (Suppl.), 121-140

8 Duprey, P. et al. (1988) Genes Dev: 2, 1647-1654

9 Desplan, C., Theis, J. and $\mathrm{O}^{\prime}$ Farrell, P.H. (1988) Cell 54, 1081-1090

10 Hoey, T. and Levine, M. (1988) Nature 332, 858-861

11 Müller, M. et al. (1988) EMBOJ.7. 4299-4304

12 Otting, G. et al. (1988) EMBO J.7, 4305-4309

13 Ko, H-S., Fast, P., McBride, W. and Staudt, L.M. (1988) Cell 55 , 135-144

14 Clerc, R. et al. (1988) Genes Dev. 2 , 1570-1581

15 Sturm, R.A., Das, G. and Herr, W. (1988) Genes Dev: 2, 1582-1599 
16 Ingraham, H.A. et al. (1988) Cell 55. 519-529

17 Bodner, M. et al. (1988) Cell 55 , 505-518
18 Wolgemuth. D.J. et al. (1989) Nature 337. 464-467

19 Balling, R., Mutter, G., Gruss, P. and Kessel, M. Cell (in press)
20 Cho, K.W.Y. et al. (1988) EMBOJ 7. 2139-2149

21 Zimmer, A. and Gruss, P. (1989) Nature 338, 150-153

\section{Autoregulation - a common property of eukaryotic transcription factors?}

\author{
EDGAR SERFLING \\ INSTITLT FL̈R VIROLOGIE IND IMMINBIOLOGIE, \\ UNIVERSTTÄT WLRZBL RG, \\ Versbacher Str. 7. D-8700 WitrzBL RG. FRG
}

The identification of homeoboxlike sequences in mammalian genes coding for tissue-specific transcription factors (see Ref. 1) suggests that many of these factors function in the same way as the proteins of homeotic genes in Drosophila, namely by establishing and maintaining the differentiation status of a given cell type. Thus, for example, one may assume that Oct-2, the B-cell-specific octamer factor controlling the expression of immunoglobulin (Ig) genes, plays an important role in B-lymphocyte development.

Numerous homeobox-containing genes in Drosophila control their own expression by positive autogenous regulation. One example among several (see Fig. 1 and Table 1) is fushi tarazu (ftz), a pair rule gene that is expressed in two phases during development: at the blastoderm stage and later during neurogenesis. The expression of $\mathrm{ftz}$ in blastoid cells is regulated by two different cis-acting DNA elements, the so-called zebra-element located next to the transcription start site and an enhancer element situated a few $k b$ further upstream ${ }^{2}$. While the activity of the zebra ( = promoter) element is controlled by the products of other pair rule and gap genes, the activity of the enhancer element is positively regulated by the $f t z$ gene product ${ }^{2}$ (Fig. 1)

At the molecular level, the $f t z$ product recognizes the sequence TCAATTAAAT located within its enhancer and autoregulates its own expression through this motif. Two other homeobox proteins, engrailed (en) and even-skipped (eve), can also bind in vitro to TCAATTAAAT sequences, but they cannot activate $f t z$ expression. Moreover, they strongly antagonize autogenous induction by $f t z^{3,4}$.

Repeats of the consensus sequences TCAATAAAT and TCAGCACCG are part of putative control regions of en and eves. When expressed in bacteria, the proteins of both genes bind to these sequences, and it is likely that en and eve autoregulate their regulatory sequences ${ }^{25}$. expression by interaction with these motifs. The proteins of the paired (prd) and zerknüllt (zen) genes have similar binding specificities (Table 1).

Homeotic genes also seem to trans-activate their own expression and that of other homeotic genes through sequence motifs composed of multiples of the trinucleotide TAA [in particular (TAA) $)_{5}$, the related sequence TAATCG, and ANNNNCATTA 5.6 . (TAA) 5 sequences, often intermingled with repeated

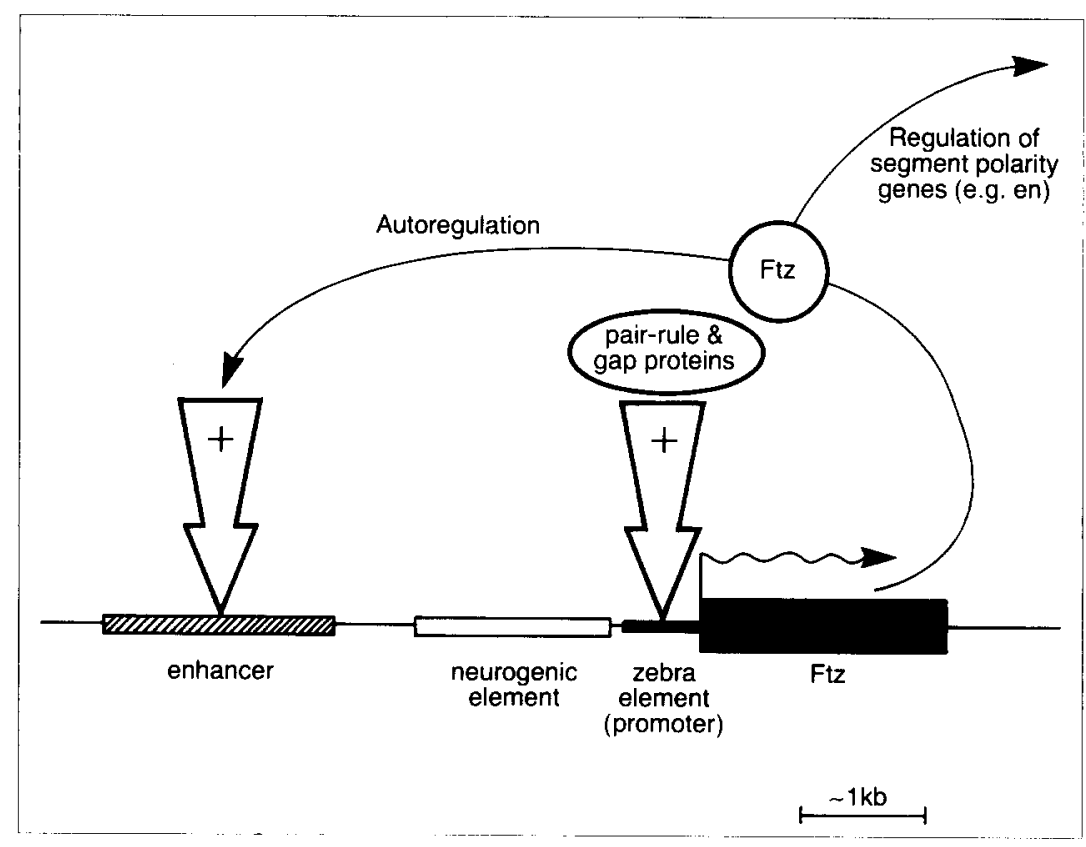

$F I G \mathbf{I}$

Scheme for autoregulation of pair rule gene fushi tarazu in blastoid cells during early Drosophila development. In blastoid cells, the expression of $\mathrm{ftz}$ is controlled both by the proximal zebra ( $=$ promoter $)$ element and the distal enhancer. While the activity of the zebra element is regulated by the products of other pair rule and gap genes, the enhancer is the target of autogenous regulation by the $\mathrm{ftz}$ gene product ${ }^{2}$. However, $\mathrm{ftz}$ can also activate other genes, such as engrailed, by direct interaction with their 